\title{
Los inicios de la enseñanza del Derecho Constitucional en el Chile decimonónico: el Liceo de Chile y el Colegio de Santiago (1828-1831)/
}

\author{
The Beginnings of the Teaching of Constitutional Law \\ in the nineteenth Century in Chile: the Liceo de Chile \\ and the Colegio de Santiago (1828-1831)
}

Rodrigo Andrés Pérez Lisicic

Universidad de Atacama, Chile

La enseñanza del Derecho Constitucional en Chile tiene su origen en dos establecimientos de educación privada: el Liceo de Chile y el Colegio de Santiago. Sus rectores eran, respectivamente, el gaditano José Joaquín de Mora y el sabio venezolano Andrés Bello y ambos colegios contemplarían los primeros programas sobre Derecho Constitucional en Chile. Pero el triunfo de los conservadores sobre los liberales (Batalla de Lircay) desarticula el centro de formación liberal más importante de la República y el ideario educativo de los conservadores, mejorando la posición de la educación pública representada por el Instituto Nacional.

Palabras Clave: José Joaquín de Mora; Andrés Bello; Liceo de Chile; Colegio de Santiago; Derecho constitucional; Derecho público.

The teaching of Constitutional Law in Chile has its origin in two private education's institutions: the Liceo de Chile and the Colegio de Santiago. Their presidents were Jose Joaquin de Mora, from Cadiz, and the Venezuelan scholar Andres Bello, respectively. Both schools included the first academic programs in Chilean Constitutional Law. The victory of conservatives over liberals, at the Battle of Lircay, disarranged the most important liberal academic institution of the Republic, and the academic project of conservatives, improving public education's position, embodied on the Instituto Nacional.

Keywords: Jose Joaquin de Mora; Andres Bello; Liceo de Chile; Colegio de Santiago; Constitutional Law; Public Law. 


\section{Introducción}

La presente investigación ${ }^{1}$ aborda el momento en el que se inicia en Chile la enseñanza formal de la asignatura de Derecho Constitucional. Con la inauguración del Instituto Nacional en 1813 — primer establecimiento del naciente Estado Nación chileno_- la enseñanza de la citada materia adquiere un lugar público con la reforma a su plan de estudios de 1832 . Durante el periodo de la Reconquista española (1814-1817), el Instituto Nacional es clausurado por decreto del general del Ejército Nacional español Mariano Osorio, un 17 de diciembre de 1814. Reinstaurado el ideario independentista del Estado chileno bajo la vigencia de la Constitución de 1818, el Instituto Nacional es reinaugurado en 1819 por el director supremo Bernardo O'Higgins, sin que, nuevamente, se haya contemplado enseñanza alguna de la Constitución chilena. Otro tanto ocurre con la existencia de las Constituciones de 1822 y 1823, respecto de las cuales no hubo iniciativa como para haber estimulado reforma alguna en el Instituto Nacional que instaurase los estudios políticos en Chile. Es curioso que un hombre ilustrado y republicano como Juan Egaña, que ostenta, entre otros honores, el haber sido el más importante ideólogo no sólo del Instituto Nacional en 1813, sino además de un proyecto de Constitución chilena hacia 1811 y padre de la Constitución de 1823, no haya propuesto la enseñanza del Derecho Constitucional patrio en los planes de estudios del principal establecimiento educacional chileno. ${ }^{2}$

Esto no deja de ser una paradoja. ¿Por qué razón está ausente la enseñanza del Derecho Constitucional en un establecimiento como el Instituto Nacional, baluarte del novel republicanismo chileno, y cuyos ideólogos -en especial Juan Egaña- constituyen, además, destacados ideólogos políticos, capaces de organizar institucionalmente al Estado en función de normas constitucionales? El Instituto Nacional no logra concretar reforma alguna para incorporar la enseñanza del Derecho Político o Constitucional en el plan de estudios jurídico. Por el contrario, sus escasas asignaturas iniciadas en 1813 siguen la inercia ininterrumpidamente mucho

1 La colaboración que se ofrece al lector constituye resultado parcial de una investigación mayor. El proyecto se intitula Orígenes de la asignatura de derecho constitucional en la enseñanza decimonónica chilena (1813-1889), código DIUDA 221154, financiado por la Dirección de Investigación y Postgrado de Universidad de Atacama, Chile.

2 Sobre la influencia de Juan Egaña en la fundación del Instituto Nacional véase Amunátegui Solar, 1889, capítulos VII, IX y X; Labarca, 1939, pp. 71 y siguientes; Silva Castro, 1953, pp. 5 y siguientes; Serrano, 1994, pp. 45 y siguientes. 
tiempo después de haberse consolidado la independencia política chilena. Hasta 1828, la enseñanza del derecho — parafraseando a Bañados Espinosa- conserva los mismos hábitos, las mismas tradiciones y el mismo estado de civilización. ${ }^{3}$

Lo que no pudo ser realizado por la educación pública, se logra en la enseñanza privada del Liceo de Chile y del Colegio de Santiago. Cada uno de estos establecimientos de educación responde a distintos proyectos políticos que entre los años 1827 y 1829 se disputan la conducción del país. José Joaquín de Mora, fundador del Liceo de Chile, pertenece a la confianza del gobierno liberal del general Francisco Antonio Pinto. El presbítero Juan Francisco Meneses, fundador y primer rector del Colegio de Santiago, y la figura de Andrés Bello, sucesor de Meneses, responden al ideario conservador de la República de Chile. Tienen la dicha estos dos centros de educación de haber inaugurado la enseñanza formal de los estudios de Derecho Constitucional.

\section{José Joaquín de Mora y el Liceo de Chile}

Con la llegada de José Joaquín de Mora (Cádiz, 1783 - Madrid, 1864) a la ciudad de Santiago un domingo 10 de febrero de $1828,{ }^{4}$ se inicia en Chile un fugaz, pero fecundo, periodo de discusiones teóricas e intensos debates políticos que giran en torno a principios que definen la esfera público-privada bajo la cual debe construirse y consolidarse la República chilena.

José Joaquín de Mora polemiza con los más distinguidos intelectuales de su tiempo en Chile: Juan Egaña y Andrés Bello. El primero defiende la institución colonial del mayorazgo cuando ésta era abolida por la Consti-

3 Bañados Espinosa, 1889, p. 2. Este profesor es el primero de la disciplina en Chile que incorpora en su texto de estudio la historia de la enseñanza del Derecho Constitucional patrio. Varias décadas después seguirán su ejemplo los profesores de la Universidad de Chile Verdugo Marinkovic y García Barcelatto, 2004, tomo I, pp. 15-19. Del mismo modo, véase Verdugo Marinkovic, Pfeffer Urquiaga y Nogueira Alcalá, 1994, tomo I, pp. 3-7.

4 Mora llega a Chile desde Buenos Aires, acompañado de su esposa Françoise Delaunneux. En 1826, el matrimonio Mora Delaunneux había arribado a la capital porteña procedente de España con la compañía del napolitano Pedro de Angelis, bajo la orden del presidente Bernardino Rivadavia. La inestabilidad política que experimentó el gobierno de Rivadavia fue la ocasión para que Pinto requiriera la presencia de Mora en Chile. En Buenos Aires dejó a su discípulo argentino Florencio Varela, con quien sostendrá una interesante correspondencia epistolar en la que se daba cuenta tanto del viaje como de la estancia de Mora en Chile y, asimismo, de las razones que justificaban su viaje a la República chilena. Sobre dicha correspondencia véase Mora, 1936, pp. 35-66; y también detalles de esa correspondencia en Amunátegui Solar, 1927, pp. 195-208. 
tución liberal de 1828.5 Andrés Bello, por su parte, traba con Mora un intenso debate acerca de literatura y educación ${ }^{6}$ justo en el momento en que se afianza políticamente el conservadurismo chileno. Al servicio del gobierno liberal de Francisco Antonio Pinto, Mora es nombrado un 11 de febrero de 1828 oficial mayor auxiliar del Ministerio de Estado. ${ }^{7}$

El ilustre gaditano es recordado por su activa participación en la redacción de la citada Constitución de 1828 y por la fundación y breve sostenimiento del Liceo de Chile. La influencia de Mora en esa Carta Magna chilena fue decisiva a propósito de las cláusulas sobre tolerancia religiosa, libertad de imprenta, no reelegibilidad del presidente de la República, bicameralidad del Congreso y abolición de los mayorazgos. En la Revista de Historia de América, número 43 correspondiente al mes de junio de 1957, el crítico literario e historiador Raúl Silva Castro sostiene como tesis que José Joaquín de Mora no constituye el más destacado ideólogo de la Constitución de 1828 y que tal error se debe al prestigioso biógrafo del gaditano Miguel Luis Amunátegui Aldunate. Abierta la polémica, no tarda en aparecer la apología de Mora efectuada por Ricardo Donoso en Cuadernos Americanos, número 100 de México, 1958, y que luego reprodujera en la Revista Chilena de Historia del Derecho, número 1, de 1959.8

5 Donoso, 1946, pp. 131-134. Este autor sintetiza las ideas expuestas por Egaña, 1828 y

6 El locus clasicus de esta polémica es Ávila Martel, 1982, el cual aporta valiosa información que no recopila Amunátegui Aldunate, 1888, como son las polémicas trabadas en El Mercurio, de Valparaíso, o El Popular, de Santiago de Chile, prensa en la que discurre el fuego cruzado entre Andrés Bello y José Joaquín de Mora sobre la legitimidad de sus respectivos establecimientos de enseñanza o acerca de la mayor o menor competencia con la que se desarrolla la enseñanza de las letras y las humanidades. De sumo interés es Poblete, 1999-2000, pp. 145-171. Este profesor del Departamento de Literatura de Universidad de California-Santa Cruz, muestra como los discursos de ambos publicistas inciden en la configuración de distintas y, a veces, contrapuestas ideas de la esfera público-privada bajo la cual se construye el Estado chileno (pp. 151-153). De gran interés son los énfasis destacados por Poblete en relación a los debates filológicos que dirige el «grupo-Bello» contra Mora y el modo cómo se produce la contra carga de éste último (pp. 155 y siguientes). El valor que ofrece el estudio del profesor Poblete reside en su tesis central: el debate sobre estilo de la lengua es de naturaleza políticosocial, pues se piensa que corrupción de la lengua e influencia de la literatura es una cuestión de corrupción de las costumbres (p. 166).

7 Amunátegui Aldunate, 1888, p. 93.

8 Silva Castro (1957, pp. 109-113) contrasta la afirmación de Amunátegui Aldunate —que tanta influencia ha ejercido en la historiografía posterior en orden a señalar la paternidad de la Constitución de 1828 al español J. J. de Mora - con las afirmaciones de Luis Galdames y, especialmente, con las del propio Mora expresadas en diversas cartas dirigidas a su amigo argentino Florencio Varela en el transcurso de 1828. Silva Castro no sólo duda de la autoría de Mora respecto de dicho texto constitucional, sino también sobre la paternidad de la redacción del periódico El Constituyente, órgano literario en el que se tiene oportunidad de comentar los avances de la Constituyente de 1828. Del mismo modo que el historiador Diego Barros Arana, Silva Castro piensa que la paternidad de tal redac- 
Fundamentalmente, la apología de Donoso respecto de la paternidad de Mora sobre la Constitución liberal chilena de 1828 descansa en la influencia que tiene el pensamiento del gaditano acerca de la libertad de imprenta, la tolerancia religiosa y la cuestión de los mayorazgos, temas tratados en la repetidamente citada Constitución. ${ }^{9}$

\section{Sobre el financiamiento del Liceo de Chile}

Esta institución constituye una obra singular en la historia de la enseñanza preparatoria y universitaria decimonónica chilena. ${ }^{10}$ Apenas instalado en el país chileno, Mora se empeña muy temprano en armar su proyecto educativo y en el periódico La Clave, números 69 y 70 de 12 y 15 de abril de 1828, informa a la opinión pública de los principios con arreglo a los cuales pretende establecer una casa de educación. ${ }^{11}$ Adelanta en este prospecto las materias a enseñar gradualmente, el uniforme que deberán vestir los estudiantes, la administración del tiempo, el régimen de castigos y premios a que da lugar el comportamiento valioso o no de los pupilos. También se anuncia el régimen de los alimentos y los fines práctico-morales de la relación alumno/profesor que ha de perseguir el comedor. Por último, agota este prospecto las condiciones económicas que permiten la manutención diaria de los estudiantes. ${ }^{12}$

ción pertenece al escritor chileno Manuel José Gandarillas. Por el contrario, la apología de J. J. de Mora es efectuada por Donoso (1959, pp. 13-24), quien opina que la convicción de Amunátegui Aldunate sobre la paternidad de la Constitución de 1828 la obtiene del escritor Ramón Briseño, 1862, quien afirma que la Constitución liberal es obra de Mora. También piensa Donoso que no es necesaria una «severa inspección» de las afirmaciones de Amunátegui Aldunate en su biografía de Mora, bastando únicamente un examen de las ideas sobre derecho público del propio Mora. Donoso utiliza las mismas fuentes empleadas por Silva Castro para sugerir la afirmación de sus tesis.

9 Donoso, 1959, p. 17.

10 La más completa recopilación documental escrita en el siglo XX sobre el Liceo de Mora pertenece a Stuardo Ortiz, 1950. Tal obra se compone de una Introducción entre las páginas 5 a 7 y el resto, de la 8 a la 146, son 101 documentos de gran interés para el conocimiento de lo que fue la génesis, desarrollo y lamentable ocaso de este destacado Liceo. El libro de Stuardo presenta un solo inconveniente: carece de índice, lo que dificulta el acceso directo a los documentos recopilados por el autor. Tratándose de las referencias bibliográficas del Liceo de Chile en el ochocientos, destaco de nuevo la biografía escrita por Amunátegui Aldunate.

11 El texto completo se encuentra reproducido en Stuardo Ortiz, 1950, pp. 9-11. El prospecto está firmado por José Joaquín de Mora el 12 de marzo de 1828.

12 En carta fechada en 11 de mayo de 1828, dirigida a Florencio Varela, Mora le expresa su real ambición: «(...) quiero formar oradores, economistas, buenos hombres públicos, que tanta falta hacen en este país, y hasta vengan profesores de Europa, tengo que desempeñar yo solo las cátedras de francés, elocuencia y economía política». Reproducido en Ibidem, p. 12. 
El Liceo de Chile es una empresa de carácter privado, pero con un significativo apoyo del gobierno liberal del vice-presidente Francisco Antonio Pinto. El 27 de agosto de 1828 Mora se dirige por escrito al citado gobernante solicitando algún edificio público carente de destino y que permitiera el funcionamiento del Liceo. Para estos efectos, fue el propio Mora quien propuso al vice-presidente que la obligación de pagar renta por el inmueble fuera retribuida con el compromiso firme de

(...) educar y mantener gratuitamente los jóvenes que V.E. designe, o lo que es lo mismo a tener en el colegio un número de becas a favor del Estado para que el Gobierno las distribuya entre los sujetos de su preferencia (...). ${ }^{13}$

En la misma fecha, el señor Pinto, con la firma de su ministro del Interior Carlos Rodríguez, decreta ceder al Liceo de Chile el edificio de $\mathrm{La}$ Maestranza, comisionándose al citado ministro para que acuerde con José Joaquín de Mora el respectivo contrato de arrendamiento. ${ }^{14}$ El 10 de septiembre de 1828 firman ambos el acuerdo legal, que contiene seis cláusulas. Destacan las dos primeras, pues se fijan diez años de arriendo del inmueble contados desde la fecha del contrato y el precio del alquiler equivalente a 2.000 pesos por diez meses, resolviéndose dicha contraprestación con diez becas de las que dispondrá el Gobierno

(...) debiendo nombrar las personas que han de obtenerlas, sin poder alterar dicho número durante los diez años estipulados. ${ }^{15}$

Pero el Liceo de Chile tendrá otras dos importantes fuentes de financiación. El gobierno de Francisco Antonio Pinto decreta el 10 de octubre de 1828 ceder al proyecto de Mora veinte becas que habían sido concedi-

13 Ibidem, pp. 13-15.

14 Ibidem, p. 15. Este autor, en nota al pie, recuerda que el edificio de la Maestranza se situaba en la vereda oriente de la actual Avenida Portugal (ex Calle de la Ollería), entre las calles Marcoleta y Rancagua, lo cual corresponde a las dependencias de la Universidad de Chile.

15 Ibidem, pp. 15-16. Con fecha 11 de septiembre el vice-presidente Pinto aprueba el contrato de arrendamiento y lo refrenda, además, con la firma del ministro de Hacienda Francisco Ruiz Tagle. El 1. ${ }^{\circ}$ de octubre de 1828 , los citados vice-presidente y ministro firman el decreto por el que designan las diez becas otorgadas por José Joaquín de Mora en retribución por el pago del precio de alquiler del inmueble cedido por el gobierno. Los beneficiarios son: Manuel y Ambrosio Rodríguez, hijos de Ambrosio Rodríguez y de Carmen Bustamante; a Gervasio, Adriano y Francisco de Paula Borgoño, hijos del brigadier José Manuel Borgoño; a Juan Esteban Campino, hijo de José Antonio Campino; a Manuel Muñoz Urzúa, hijo de Manuel Urzúa y de Tomasa Gamero; a Francisco y Pedro de la Lastra, hijos del brigadier Francisco de la Lastra; y a Narciso Guerrero, hijo de Ramón Guerrero» (p. 17). 
das por un decreto de 28 de noviembre de 1827 en favor del ciudadano José Melián, quien proyectaba establecer un colegio para niños en la ciudad de Santiago. Tal empresa del señor Melián había fracasado y en aquel decreto de 1827 , el gobierno no sólo disponía proveer veinte becas a distribuir entre las ocho provincias del país, sino que ordenaba a la Tesorería General pagar 4.000 pesos «(...) para el transporte de los profesores que han de traerse de Europa». Esos cuatro mil pesos, en el decreto de 1828, se destinaron al propósito de ayudar a los gastos «(...) en las primeras atenciones del establecimiento» de José Joaquín de Mora. ${ }^{16}$

Sin embargo, el más importante apoyo económico al Liceo de Chile tuvo como fuente el propio Congreso Nacional. El 11 de noviembre de 1828, el Ejecutivo dirigía al Senado del Congreso Nacional un proyecto de ley en el que se solicitaba la autorización para conceder cuarenta y dos becas al Liceo de Chile. El presidente del Senado respondió al vice-presidente Pinto concediendo las cuarenta y dos becas al Liceo de Chile. ${ }^{17}$ Pero agregaba algo más la nota parlamentaria. Si bien el Congreso está consciente de la importancia de apoyar a iniciativas que, como el Liceo de Chile, cultiven el amor y verdadera adhesión a la ley entre los ciudadanos, esperaba del gobierno no sólo una reforma del Instituto Nacional, sino también igual actitud benéfica para con el primer establecimiento público de enseñanza nacional. ${ }^{18}$

16 El decreto de 28 de noviembre de 1827 aparece reproducido en nota al pie número 4 en Ibidem, p. 18. Las veinte becas dispuestas por el gobierno para estudiantes de las provincias se distribuyeron así: dos para cada una de las provincias de Chiloé, Valdivia, Concepción y el Maule; tres para cada una de las provincias de Colchagua, Santiago, Aconcagua y Coquimbo. El decreto firmado por Pinto y Rodríguez es también refrendado por Francisco Ruiz Tagle, ministro de Hacienda.

17 Como anécdota, el presente investigador no puede obviar un dato de relevancia para la cultura atacameña. Entre los estudiantes favorecidos con una de las cuarenta y dos becas se encuentra el gran escritor costumbrista, filósofo y poeta José Joaquín Vallejo, Jotabeche, destacado alumno del Liceo. Su nombre es propuesto al vice-presidente Pinto por el diputado de la provincia de Coquimbo Buenaventura Marín. El Ejecutivo, a través del ministro Rodríguez, notifica al director del Liceo de Chile un 26 de marzo de 1829 que el vice-presidente otorga la gracia «al joven Joaquín Vallejo». Véase Stuardo Ortiz, 1950, pp. 49-50.

18 Es una opinión frecuente entre los estudiosos de la enseñanza decimonónica chilena que la protección dada por el gobierno de Pinto al Liceo de Chile fue en claro desmedro del Instituto Nacional. En este sentido lo trata Ávila Martel (1982, p. 24), agregando que tal protección al Liceo creo un núcleo de oposición a aquel establecimiento que, como recuerda también Serrano (1994, p. 51), provoca la ardiente oposición del presbítero Meneses, rector del Instituto Nacional, que junto al sector conservador, fundan el Colegio de Santiago. También Labarca (1939, p. 84), sostiene la desprotección en que había quedado el Instituto Nacional con la coexistencia del Liceo de Chile y el Colegio de Santiago entre 1828 y 1831 . 


\section{Sobre los estudios generales y el curso de derechos en el Liceo de Chile}

En el transcurso de 1828, Mora publica su Plan de estudios del Liceo de Chile. Es el texto consultado en la presente investigación con el fin de conocer las materias ofertadas para la enseñanza general que imparte Mora en su Liceo de Chile. Este establecimiento educacional abrió sus puertas un 16 de enero de $1829,{ }^{19}$ pese a que el prospecto indicaba que su apertura fue el $1 .^{\circ}$ de enero de aquel año. ${ }^{20}$

Dos son las áreas de conocimientos que el citado Liceo propone a los estudiantes: estudios literarios y estudios científicos y la duración de estas enseñanzas sería de cinco años. Los estudios literarios estaban a cargo de José Joaquín de Mora y se realizaban por la mañana; los estudios científicos, a cargo de Andrés Gorbea, tenían lugar durante la tarde. El plan de estudios era el siguiente: ${ }^{21}$

\section{Primer año.-}

Estudios Literarios bajo la dirección de don José Joaquín de Mora.

Por la mañana. Gramática latina. Traducción francesa por el sistema de Hamilton. Dos veces por semana, Geografía descriptiva.

Estudios Científicos bajo la dirección de don Andrés Gorbea.

Por la tarde. Aritmética, Álgebra, Geometría especulativa y Trigonometría rectilínea; una vez por semana, en el último semestre, lección y práctica de la Geometría aplicada.

\section{Segundo año.-}

Gramática latina. Gramática francesa y continuación de la traducción por el sistema de Hamilton. Dos veces por semana Historia sagrada, y de los imperios antiguos.

Secciones únicas; cálculos de combinaciones, permutaciones y probabilidades; ecuaciones superiores; series; Trigonometría esférica; uso de los globos; Geometría aplicada a las tres dimensiones.

\section{Tercer año.-}

Sintaxis latina aplicada a la lectura de los autores. Literatura francesa. Dos veces por semana. Historia Griega.

Cálculo diferencial, integral y de las variaciones; Estática, Dinámica; Fortificación pasajera y permanente.

19 Baeza Marambio, 1944, p. 59.

20 Plan de estudios del Liceo de Chile, con algunos pormenores sobre su ejecución y sobre la disciplina del establecimiento, 1828 , p. 1.

21 Ibidem, pp. 1-2. 


\section{Cuarto año.-}

Lectura razonada de autores latinos. Gramática castellana analizada. Dos veces por semana Historia Romana; dos veces por semana en los últimos seis meses, Economía Política.

Hidrostática, Hidrodinámica, Magnetismo; Óptica y Principios de Astronomía y Perspectiva.

\section{Quinto año.-}

Lectura razonada y análisis de autores latinos, españoles y franceses. En los seis primeros meses Elocuencia castellana, y literatura española; en los otros seis Ideología. Dos veces por semana, Épocas de la Historia Moderna, según los sistemas de Koch y de Müller.

Química; cuerpos inorgánicos, cuerpos organizados vegetales y animales; Análisis químico.

De dicho programa no se infiere un estudio sistemático de la política y de sus instituciones más relevantes. Al momento de explicarse con detalle cuál es el alcance de los «Estudios literarios», el director del Liceo José Joaquín de Mora afirma:

En una escuela práctica proporcionada a sus conocimientos, tendrán frecuentes ocasiones de aplicar las teorías que hayan aprendido. No sólo compondrán discursos escritos sobre puntos de moral, de gusto y de historia, sino que discutirán entre sí cuestiones de Derecho y de política, aprovechándose a veces de las ocasiones que les suministren los trabajos de la legislatura nacional. Esta práctica, común en las universidades inglesas, ha sido el semillero de muchos grandes oradores y eminentes hombres públicos. ${ }^{22}$

Por otra parte, José Joaquín de Mora organizó un curso general de derechos, el cual difunde a través de un prospecto que circuló durante el mes de abril de 1829. Las materias o asignaturas del citado curso se distribuían en tres años $\mathrm{y}$, en dicho plan, la enseñanza del Derecho Constitucional — que he destacado en cursiva - se inicia en el primer año de los estudios. Véase el contenido del prospecto a continuación: ${ }^{23}$

Se abrirá en el Liceo de Chile un curso general de derechos bajo la inmediata dirección de don José Joaquín de Mora. Durará tres años, en los cuales se distribuirán del modo siguiente los diferentes ramos de esta enseñanza:

22 Ibidem, p. 4.

23 El programa del curso general de derechos se encuentra en el Capítulo VIII de la obra de Amunátegui Aldunate, 1888, pp. 171-172. Un extracto del prospecto del curso general de derechos de Mora, que contiene sólo las materias o asignaturas, se encuentra en Baeza Marambio, 1944, p. 59, pero adolece de descuido en la cita del programa. 
Primer año.- Derecho Natural, de Gentes, Constitucional y Romano, sirviendo de texto a las explicaciones del profesor, las obras de Burlamachi y Vattel; la Constitución de la República y la Instituta de Justiniano. Para todo lo relativo al Derecho Internacional y Marítimo, se hará uso de las doctrinas de Azuni y Peuchet, que se miran en las naciones cultas como oráculos de esta parte de la legislación.

Segundo año-- Derecho Patrio o explicación de todas las leyes civiles y criminales que rigen en nuestro actual sistema judiciario comparándolos con los que han adoptado los pueblos más célebres e importantes de la época presente.

Tercer año. Derecho Canónico y Economía Política. El primero será un extracto de lo más puro que encierra una ciencia extraviada de su verdadero objeto por el espíritu escolástico, y un resumen de las disposiciones más aplicables a las instituciones políticas que nos rigen. La economía política se enseñará por el tratado de Jacobo Mill, añadiéndose lo más sensato y positivo que comprenden los otros economistas que han escrito antes y después.

Como el objeto de este curso es formar letrados útiles, se empleará en él la lengua nacional, que es la que se usa en los tribunales de nuestro país. El curso de derechos será el único del Liceo en que se admitan externos; y para cortar toda comunicación entre éstos y lo interior de la casa, se tendrá la clase en las piezas exteriores que antes ocupó la capilla de Loreto. Los externos pagarán cuatro pesos mensuales. Los jóvenes que entren al Liceo en calidad de alumnos internos para seguir el curso de derechos, podrán tomar parte en los otros ramos de instrucción que se cursan en el establecimiento.

Los propósitos que Mora tenía en mente en relación al curso de derechos eran de orden superior, que en absoluto se agotaban con la enseñanza al interior del Liceo. El gaditano concibió su curso de estudios jurídicos como Curso de derechos del Liceo de Chile, aplicable a todas las nuevas repúblicas de América, ${ }^{24}$ resolviendo la publicación de una obra que permitiera su difusión. Mora decide publicar siete tomos o cuadernos del curso de derechos: El primer tomo se destina a la enseñanza del Derecho Natural y de gentes; el segundo, a la enseñanza del Derecho Romano; el tercer y cuarto tomos, reunidos, versaban sobre Derecho Civil y Criminal; el quinto estaba pensado para la enseñanza del Derecho Comercial; el sexto tomo, dedicado a la enseñanza del Derecho Canónico; por último, el séptimo se concibió para la enseñanza de la Economía Política y del Derecho Constitucional. La Clave de Chile contiene una breve descripción de las materias del tomo 7. ${ }^{\circ}$ sobre Economía Política y Derecho Constitucional..$^{25}$

24 Ver Mora, 13 de agosto de 1829, p. 34, acompañando a estos efectos el índice de materias de cada uno de los siete tomos. Se ha tenido a la vista el original que consta de cuatro páginas, numeradas de la 31 a la 34, el que se cita junto con la referencia recopilada por Stuardo Ortiz, 1950, pp. 61-65.

25 Ibidem. 


\section{Tomo $7 .^{\circ}$}

\section{Economía política y derecho constitucional}

Primera parte. Exposición del tratado de economía política de Mill, con las doctrinas

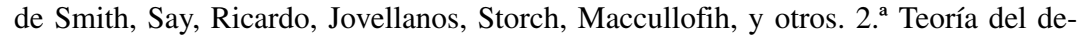
recho Constitucional, aplicada a la Constitución de Chile. Apéndice.- Ensayo de un curso de lógica legal, o reglas de raciocinio en materias de legislación y de jurisprudencia.

A diferencia de los extensos descriptores que Mora dedica a otras materias como el Derecho Natural, el Derecho de Gentes, o el Derecho Civil y el Derecho Penal, el de Derecho Constitucional es extremadamente pobre, como se puede apreciar. Tampoco ha sido de utilidad recurrir a una rica fuente de información de la época como son los programas de exámenes, en los que se estilaba informar a los estudiantes del temario de cada asignatura sujeta a evaluación final. De este modo, en el Programa de los exámenes públicos en el Liceo de Chile en los días 8, 9 y 11 de febrero de 1830 no se halla referencia alguna a los contenidos de Derecho Constitucional. Únicamente se detallan los contenidos a evaluar de las asignaturas de Derecho Natural y Derecho de Gentes, señalando los alumnos que «responden» tales materias: Buenaventura Cousiño, Aniceto Cordovez, José Rojas, Manuel Guzmán, Manuel Quiroga, Juan Nicolás Álvarez, Joaquín Bravo, Joaquín Álamos, Fernando Herrera, Diego Tagle, José Antonio Valdés, Manuel Rojas y Manuel Allende. ${ }^{26}$

Si el Derecho Constitucional y el Derecho Romano son materias del primer año de estudios del curso especial de derechos, ¿por qué razón no aparecen en la convocatoria de febrero junto al Derecho Natural y al Derecho de Gentes? Es posible que tales materias no se hubieran enseñado a tiempo como se tenía previsto, no habiendo razón para evaluarlas en la fecha de exámenes fijada por el Liceo de Chile.

La obra es publicada parcialmente. Los suscriptores del primer tomo recibirían su distribución a partir del $1 .^{\circ}$ de enero de 1830. Sin embargo, hubo que esperar hasta el $1 .^{\circ}$ de abril de aquel año para ver el cuaderno publicado. El segundo tomo, sobre Derecho Romano, logra su publicación mucho más tarde y, según Miguel Luis Amunátegui, la obra se usó en tres universidades bolivianas hasta 1865. Los cinco restantes cuadernos no se publican, con lo que jamás se podrá saber el contenido y orientación de los

26 Stuardo Ortiz, 1950, pp. 67-82. El temario de la asignatura de derecho natural se encuentra en pp. 67-69, y el de derecho de gentes en pp. 69-71. 
estudios del Derecho Constitucional pensado por Mora. ${ }^{27}$ No cabe duda que los acontecimientos políticos - la derrota de los liberales en la batalla de Lircay en 1829 - restan a Mora la tranquilidad requerida para la conclusión editorial del curso de derechos.

\section{Colegio de Santiago: Antecedentes}

El Colegio de Santiago se funda en la necesidad del sector conservador de oponer resistencia a la empresa educativa y liberal de Mora y apoyada por el propio gobierno de Pinto, en desmedro - como se dijo- del Instituto Nacional. Se trata de una empresa privada, financiada por el mercader y siniestro Diego Portales Palazuelos,$^{28}$ quien logra reunir, con el esfuerzo e impulso inicial de Pierre de Chapuis, a un distinguido grupo de profesores de origen francés. Chapuis los reclutó en Francia - donde forma la Sociedad Educacional- y en Brasil y llegaron al puerto de Valparaíso el 8 de diciembre de 1828, a bordo del buque de la marina francesa $L$ 'Adour. ${ }^{29}$ Unos días más tarde, Chapuis publica un Aviso en la Gaceta de Chile número 12, donde da cuenta sobre los antecedentes del grupo contratado en París y de cuáles son las bases de la sociedad educacional que ha de regir en Chile. A través de dicho documento se conocen los nombres de los profesores miembros de esa sociedad parisina. Estos eran: Juan Antonio Portes, doctor en letras humanas, profesor de filosofía y de economía política; Claudio Gay, doctor en ciencias, profesor de física, química e historia natural; José Coupelon, bachiller en letras humanas,

27 Amunátegui Aldunate, 1888, p. 174.

28 Diego Portales Palazuelos destaca por sus tácticas maquiavélicas en política, antes y después de haber obtenido el poder tras la Batalla de Lircay, en la que triunfan los conservadores para derrocar y despojar del gobierno a los liberales — civiles y militares - leales al ex vicepresidente Francisco Antonio Pinto. El 30 de julio de 1830, en pleno apogeo del gobierno conservador, expulsa del país a su amigo liberal Pierre de Chapuis. Véase al respecto Ávila Martel, 1982, p. 28. Una mirada atenta y crítica, que revela los horrores ético-políticos de Portales y su obra, se encuentra en el historiador chileno Salazar Vergara, 2007, p. 25, donde el autor sostiene que el orden portaliano no responde a los intereses ni de las clases productoras, ni de las clases asalariadas. Especialmente véase de la obra de Salazar Vergara su «Capítulo VI Culminación de los procesos revolucionario y contrarrevolucionario (1828-1837)», pp. 315-412, donde el autor reconstruye históricamente la red de ardides del estilo político portaliano, concebido para fomentar la represión policial y carcelaria, como asimismo para la creación de leyes secretas que autorizan la existencia de policías secretas y ejecuciones políticas sin juicio previo.

29 En el Capítulo III de Ávila Martel, 1982, pp. 27-30, se encuentra una referencia con mayores detalles sobre la empresa educacional de Chapuis. 
profesor de retórica, literatura griega, latina y de geografía; Luis Teodoro Morinière, bachiller en letras humanas, licenciado en Leyes y profesor de bellas letras, de derecho romano, derecho natural y derecho de gentes, además de historia; por último, Francisco de Lubin Gillet de Laumont, profesor de mineralogía y matemáticas. ${ }^{30}$

Hacia el mes de febrero de 1829, Pierre de Chapuis es apartado de la empresa educacional. En su reemplazo, Diego Portales incorpora al presbítero Juan Francisco Meneses, quien a la sazón era rector del Instituto Nacional. Este presbítero fue el primer rector del Colegio de Santiago, correspondiéndole su instalación y apertura el 16 de marzo de 1829. Durante su breve rectorado no existe fomento alguno para la enseñanza de los estudios políticos o constitucionales. Hay que tener en cuenta que si Meneses no había promovido esos estudios siendo rector del Instituto Nacional, no existía razón plausible para que los impulsara ocupando el mismo cargo máximo en el Colegio de Santiago, al cual accedió más bien por los estrechos vínculos de amistad que mantenía con Diego Portales, que por el interés de innovar significativamente la enseñanza chilena.

En diciembre de 1829, el rector Meneses acepta participar en el gobierno conservador ${ }^{31} \mathrm{y}$, poco tiempo después, resulta elegido rector de la Universidad de San Felipe, cargo que desempeña hasta el día de la extinción de esta Casa de Estudios en 1838. Hacia 1831 asume también la función de secretario de la Convención que redacta la Constitución de 1833. Posteriormente, luego de iniciadas las actividades de la Universidad de Chile en 1842, Meneses asumiría el decanato de la Facultad de Leyes en 1846, ocupando este puesto hasta el año de su muerte ocurrida en 1860.

\section{Los estudios generales en el Colegio de Santiago}

El Colegio de Santiago inició sus actividades en marzo de 1829, disponiendo su rector Meneses un Reglamento interior y un Cuadro de ejer-

30 El Aviso aparece publicado el 31 de diciembre de 1828. Reproducido por Ávila Martel, 1982 , p. 56.

31 Juan Francisco Meneses fue secretario general de la Junta de Gobierno integrada por José Tomás Ovalle (presidente), Isidoro Errázuriz y José María Guzmán. El decreto de nombramiento de su nombramiento es de 8 de enero de 1830; sin embargo, actuó de hecho desde la fecha misma de instalación de la citada Junta, ocurrida el 24 de diciembre de 1829. Véase Valencia Avaria, 1986, p. 465. 
cicios para los alumnos del establecimiento. ${ }^{32}$ El Reglamento tenía el sello personal del presbítero, pues partía como punto de inicio de la máxima de que la «religión es la base esencial de toda buena educación», determinándose a partir de este principio los ritos oratorios al inicio y término de cada clase, así como el contenido que debía observar la clase elemental y todas las demás de aquellos alumnos que estudiaban gramática: historia del viejo y nuevo testamento, en la primera; y disertación de memoria de algunos versículos en latín de la historia sagrada, en las segundas. Se agrega a esta inspiración el compromiso del rector Meneses en dictar los sábados de cada semana las explicaciones sobre algún punto de la doctrina cristiana. En lo demás, el reglamento interior del Colegio de Santiago establecía obligaciones muy estrictas para los alumnos, ${ }^{33}$ un régimen de castigos y premios y de disposiciones generales que versaban nuevamente sobre conducta estudiantil y relación de los profesores con las familias de los alumnos.

Por otra parte, el cuadro de ejercicios ordena el horario de estudio de cada materia o asignatura, que se extiende desde las siete de la mañana hasta las diecinueve y treinta minutos, de lunes a sábado.

Sobre los estudios generales que ofrece el Colegio de Santiago, es necesario determinar dos momentos. El primero de ellos, corresponde al plan de estudios bajo el rectorado de Juan Francisco Meneses. El segundo momento, se vincula al plan de estudios que Andrés Bello propone para el Colegio de Santiago apenas iniciado su periodo rectoral.

El rectorado de Meneses se inicia con un plan de estudios que la sociedad de profesores del Colegio de Santiago da a conocer a la opinión pública durante el mes de enero de 1829, intitulado Prospecto del Colegio de Santiago ${ }^{34}$ al que adicionan un Cuadro de ejercicios para cada día de la semana. Éste último es distinto del que ordena publicar Meneses el 11 de marzo de 1829 y que, a diferencia del anterior, no contempla la enseñanza de la mineralogía. En todo lo demás, se corresponde con las materias contempladas en el prospecto del mes de enero, cambiando únicamente el horario y organización de los estudios.

32 Reglamento interior del Colegio de Santiago, 1829. Lo firma Meneses con fecha 11 de marzo de 1829. Reproducido por Ávila Martel, 1982, pp. 75-76. El Cuadro de ejercicios para cada día de la semana, 1829, anexo al Reglamento anterior, aparece reproducido por Ávila Martel, 1982, pp. 76-77.

33 Verbi gratia, el modo como los estudiantes entran y salen de sus salas de clases, reglas sobre decencia en el vestir y limpieza corporal rigurosa, o reglas sobre el deber de sumisión, respeto y perfecta obediencia a los profesores.

34 Prospecto del Colegio de Santiago, 1829. Reproducido por Ávila Martel, 1982, pp. 59-68. 
El Prospecto de enero de 1829 divide la enseñanza en tres áreas de formación: la enseñanza elemental, la enseñanza de las humanidades y bellas letras y, por último, la enseñanza de las ciencias.

La primera - de nivel elemental - comprende la historia sagrada, gramática latina, geografía elemental, aritmética, escritura y dibujo. Todo ello en un primer año. En el segundo año, se ejercitan los estudiantes en la traducción de autores latinos, iniciándose en el estudio de la lengua griega. Complementan su formación en esta fase con la continuación de sus lecciones de aritmética, dibujo y de escritura. En el tercer año, se inicia la formación en las humanidades y bellas letras, que se prolonga hasta un cuarto año. En su aplicación, durante el primer año de estas humanidades se explican las obras de Quinto Curcio, Tito Livio, Virgilio y Ovidio; también las fábulas de Esopo y el nuevo testamento en griego. Al mismo tiempo, continúan sus lecciones de aritmética y de dibujo, cesando las clases de escritura. En el segundo año de las humanidades (cuarto año de los estudios) los alumnos aprenden las obras de Salustio, Tácito, Cicerón, Horacio, Luciano, Jenofonte y Homero. Destaca en esta fase la enseñanza de la retórica, a través de la composición de narraciones en latín y en español. Para estos efectos, se continúa con la enseñanza de la versificación latina y del dibujo.

Finalmente, el Prospecto de enero de 1829 detalla la enseñanza de las ciencias. Los saberes que se incorporan al estatus de las ciencias en el plan de estudios son la filosofía, la economía política, el derecho romano, las matemáticas, la geografía, la historia, la historia natural y la física junto a la química. El Cuadro de ejercicios del Colegio de Santiago rotula a estas materias como «cursos especiales».

La filosofía comprende la metafísica, la lógica y la moral. Respecto de la economía política, el Prospecto exige que se estudien algunas verdades sobre esta ciencia obtenidas por diversos autores a lo largo de la historia como Platón, Aristóteles, Quesnay, Montesquieu, Condillac, entre otros, para luego abordar el sistema de economía que proponen Jean Baptiste Say y Adam Smith, «los únicos que han hecho de esta ciencia una doctrina completa», según reza el prospecto.

La enseñanza del derecho romano se limita a la exposición de su historia, su legislación bajo los reyes, los cónsules y los emperadores, abrazando la compilación de Justiniano para enseguida comparar esa legislación romana con la moderna de algunos pueblos.

En cuanto a las matemáticas, se divide su enseñanza en tres años. Durante el primero de ellos, se abarcan la aritmética, el álgebra, la geome- 
tría y la trigonometría. Durante el segundo año, finaliza la enseñanza del álgebra, se inicia su aplicación a la geometría y culmina con el estudio de la estadística. El tercer año se destina a la enseñanza del cálculo integral y diferencial.

En relación a la geografía, se explican los fenómenos que derivan del movimiento de los cuerpos celestes, para en seguida abordar el estudio de las costumbres, los usos, la religión y la política de los diferentes lugares que se describen en la asignatura. De este modo, la geografía se divide en física, moral y política. Se agrega a esta enseñanza la demostración de la confección de las cartas marítimas más exactas del momento.

La enseñanza de la historia comprende la historia antigua (origen de las sociedades humanas, cultura egipcia, asiria, griega y romana hasta su declinación), la historia del bajo imperio o Edad Media (los tiempos de Constantino y la grandeza de Bizancio) y la historia moderna (desde Carlomagno hasta el estudio particular de la historia de los pueblos más considerables de Europa). Por otra parte, la enseñanza de la historia natural abraza tres disciplinas como son la zoología, la botánica y la mineralogía.

Concluye la formación científica con la enseñanza de la física y de la química. La primera se enseña en los cinco primeros meses del año; la química, en los meses restantes.

En el próximo apartado de esta investigación se explica el contenido del plan de estudios que adopta el Colegio de Santiago el año en que Andrés Bello asume su rectorado. En ese nuevo plan aparece la enseñanza del Derecho Constitucional.

\section{El estudio del Derecho Constitucional en el nuevo plan de estudios del Colegio de Santiago}

Los estudios políticos se iniciaron bajo el rectorado de Andrés Bello, con la denominación Ciencia de la Legislación. El 2 de marzo de 1830, aparece publicado el Prospecto del Colegio de Santiago, con las firmas de Andrés Bello (director del Colegio) y los profesores societarios $\mathrm{H}$. Beauchemin, J. Coupelon, Claudio Gay y Théodore Morinière, fechadas el $1 .^{\circ}$ de febrero de $1830 .{ }^{35}$ Bello no sólo asumía el cargo de director, sino que

35 Ibidem, pp. 88-94, donde ofrece el Prospecto íntegro. 
también trabajó como profesor de las asignaturas de Lengua y Literatura Castellana y la de Legislación.

Los estudios generales bajo el nuevo plan de 1830 se organizaban en tres áreas de conocimiento. En primer lugar, las llamadas «Letras y Bellas Artes», que comprendían gramática castellana (en un año), gramática latina (en tres años), latinidad superior (en dos años), retórica (en un año), lengua francesa (en dos años) y lengua y literatura castellana (un año). En segundo lugar, se ofrecía un área de «Ciencias», incluyendo filosofía (un año), legislación (un año), matemáticas (un primer año de aritmética y un segundo de álgebra y geometría), geografía (un año dividido en dos secciones, donde la última de ellas se componía de cuatro ramos) e historia natural (un año repartido en tres partes, zoología, botánica y mineralogía). En tercer lugar, el Colegio de Santiago ofrecía cursos de «Bellas Artes», comprendiendo arte de escribir (materia que pueden cursar los estudiantes de las clases de latinidad), dibujo y música. Además de estos estudios principales, el Colegio de Santiago otorgaba «una importancia particular» a la instrucción religiosa, la que, según se indica, se consagraba el sábado por la tarde de cada semana, a cargo del capellán del Colegio, quien daba las lecciones «sobre las verdades fundamentales de la religión, y sobre la historia sagrada». Por último, el Colegio de Santiago contemplaba una «Escuela de Primeras Letras», a cargo de los señores Domingo Acevedo y Pedro Douet, donde enseñaban a leer, escribir, contar y catecismo.

Tratándose de la asignatura de Legislación o Ciencia de la legislación, el folletín in comento entrega un descriptor de sus contenidos, como se observa a continuación.

En este curso se explicará la teoría de la legislación, reduciendo a un cuerpo de doctrina la de las obras del ilustre Bentham, sin perder de vista lo que se ha escrito sobre este interesante asunto por Filanguieri, Beccaria, Montesquieu, Benjamin Constant y otros célebres publicistas de nuestros días. El principio de utilidad, desenvuelto con precisión, conducirá a la análisis del bien y el mal político, a la refutación de los sofismas que suelen oscurecer la discusión de las materias legales, y al examen de algunas máximas abstractas, cuya exageración o errada inteligencia ha extraviado no pocas veces el espíritu de reforma, y malogrado las revoluciones. A la luz del mismo principio se observarán los elementos de los varios sistemas constitucionales, su espíritu y tendencia; se examinarán los derechos y obligaciones civiles; se establecerán las máximas reguladoras del código penal, de la constitución de los juzgados para la averiguación de los hechos y para la imparcial aplicación de las leyes; y en fin, de la economía política y de la administración fiscal. Estas materias se explicarán y discutirán verbalmente, y se concentrará la sustancia de ellas en un resumen dialogado, que se dictará por escrito. 
Andrés Bello es el fundador en Chile de esta asignatura de clara influencia benthamiana. Está compuesta de materias como la teoría del Derecho o de la justicia, el Derecho Político o Constitucional, el Derecho Civil, el Derecho Penal y la Economía Política. El programa de esta materia es el antecedente inmediato de la asignatura de Legislación Universal, que inaugura el Instituto Nacional a través de su plan de estudios de 1832. También allí Andrés Bello será su profesor. Para esa fecha este famoso polígrafo integra en la asignatura sólo el Derecho Civil, el Constitucional y el Penal.

Las materias correspondientes al Derecho Constitucional de aquella asignatura de ciencia de la legislación se encuentran en el Programa para los exámenes de los alumnos de la clase de principios generales de legislación, de 1831, del Colegio de Santiago. El Programa convoca a estos exámenes a cinco estudiantes: J. Domingo Aguirre, Pedro Nolasco Cruzat, Vicente Bascuñán, J. Ignacio Errázuriz y J. Manuel Errázuriz. ${ }^{36}$ Al Libro II del Programa Andrés Bello le denomina Derecho político, aunque se trata más bien de un tradicional programa de derecho constitucional. Consta de diez apartados o unidades temáticas. La primera de ellas se intitula Origen de los gobiernos; la segunda, Causas que mantienen de hecho la sumisión de los súbditos al gobierno; la tercera, Razón en que se funda la obligación de obedecer al gobierno; la cuarta unidad se denomina Libertad civil; la quinta, Diferentes especies de gobierno; la sexta, Soberanía del pueblo; la séptima unidad, De la legislatura; la octava, Poder ejecutivo; la novena unidad temática, Federaciones y, por último, la décima unidad se denomina De la opinión pública.

\section{Conclusiones}

La enseñanza del Derecho Constitucional chileno surge de los proyectos de educación privada liberal y conservador. El primero de ellos, representado por la figura de José Joaquín de Mora y la instalación de su Liceo de Chile; el segundo, financiado por el mercader Diego Portales Palazuelos, gira en torno a dos personalidades como son Juan Francisco

36 El Programa para los exámenes del Colegio de Santiago aparece publicado como prospecto por la Imprenta de la Opinión, en 1831. El texto que se utiliza en esta investigación es el compilado por Ávila Martel, 1982, pp. 96-109. Consta el prospecto de cuatro libros: principios generales (p. 96), derecho político (p. 100), de los derechos y obligaciones civiles (p. 104) y delitos y penas (p. 106). 
Meneses y Andrés Bello, los rostros institucionales del Colegio de Santiago.

La incorporación de la asignatura de Derecho Constitucional en los planes de estudio de la enseñanza privada adquiere carta de ciudadanía de manos de Mora y de Bello. Esto es una paradoja de la cultura jurídico-política chilena, pues habiendo una praxis política y un conocimiento de las formas constitucionales desde los primeros años de la República, los protagonistas de dichos procesos políticos se muestran indiferentes en relación a la enseñanza de la Constitución al momento de decidir acerca de los contenidos relevantes para la formación jurídica de los juristas. La influencia de un intelectual como Juan Egaña se concentra más bien en la redacción de textos constitucionales que en la enseñanza jurídica del derecho de la Constitución. Existe un divorcio entre el proceso político tendiente hacia la consolidación de un gobierno independiente - preocupado de la constitucionalización de la actuación política — y la necesaria ideologización del pueblo en torno al valor político de una Constitución.

En esto radica la importancia del aporte de Mora y Bello, que fractura ese divorcio entre política y enseñanza de la política, el divorcio entre Constitución y enseñanza del derecho de la Constitución.

Si la hipótesis de investigación es que las fuerzas políticas liberales hacia 1828 tenían como propósito enfatizar, a través de la enseñanza del Derecho Constitucional, los derechos del pueblo, por una parte, y por la otra, que las fuerzas políticas conservadoras hacia 1829 planteaban realzar, también a través de la enseñanza del derecho de la Constitución, los derechos del soberano o del gobierno, entonces no resulta del todo posible responder a tal hipótesis a partir de los materiales investigados. La razón de ello es la ausencia de un programa de estudios de Derecho Constitucional en el Liceo de Chile, o bien de un programa de contenidos convocados para el examen final de la asignatura. Lo único disponible son escuetas referencias contenidas en el plan de estudios del Curso de derechos del Liceo de Chile y en la propuesta de contenidos del libro séptimo del mismo curso, que nunca se publicó. En aquel plan de estudios, únicamente se establece el texto de la Constitución de la República para la enseñanza del Derecho Constitucional. En cuanto al contenido del libro séptimo del Curso de Derechos, se afirma que la enseñanza del Derecho Constitucional se arregla conforme a la teoría constitucional aplicada a la Constitución de la República. Con el pesar del presente investigador, es una pérdida para la tradición liberal chilena el que Mora no haya explicitado su metodología y 
dogmática del citado Derecho Constitucional en los planes y programas del Liceo de Chile. No es suficiente saber que la obra de Jeremy Bentham influye en la doctrina constitucional del gaditano pues Andrés Bello también la tiene, además de la que recibe de Benjamin Constant, como él mismo declara. En este sentido, Bello es más cuidadoso que Mora, toda vez que logra informar sobre los contenidos de la enseñanza del derecho constitucional en el prospecto publicado para la realización de los exámenes finales del Colegio de Santiago, junto al de todas las demás asignaturas. No ocurre lo mismo con el prospecto de esta clase de exámenes del Liceo de Chile, en la que queda excluida la prueba o examen final del Derecho Constitucional o, lisa y llanamente, nada se dice sobre el particular. ¿Qué debemos pensar? ¿Qué Mora jamás enseñó Derecho Constitucional en el año de 1829? ¿O que simplemente omite el gaditano transcribir en el prospecto la convocatoria para los exámenes finales de esa materia? A diferencia de lo que ocurre con la asignatura de Derecho Constitucional, respecto a las de derecho romano y derecho de gentes, Mora entrega a los estudiosos de la historia de la enseñanza del derecho una valiosa información, rica en contenidos y en la adopción de tradiciones teóricas.

Recibido el 16 de enero de 2010 Aceptado el 25 de marzo de 2011

\section{Bibliografía}

Amunátegui Aldunate, Miguel L.: Don José Joaquín de Mora: Apuntes biográficos, Santiago de Chile, Imprenta Nacional, 1888.

Amunátegui Solar, D.: Los primeros años del Instituto Nacional, Santiago de Chile, Imprenta Cervantes, 1889.

Amunátegui Solar, «Recuerdos de don José Joaquín de Mora», Studium, 3, Santiago de Chile, 1927, 195-208.

Ávila Martel, Alamiro de: Mora y Bello en Chile (1829-1831), Santiago de Chile, Ediciones de la Universidad de Chile, 1982.

Baeza Marambio, Mario: Esquema y notas para una historia de la Facultad de Ciencias Jurídicas y Sociales de la Universidad de Chile, Santiago de Chile, Universidad de Chile, 1944.

Bañados Espinosa, Julio: Derecho constitucional. Constituciones de Chile, Francia, Estados Unidos, República Argentina, Brasil, Bélgica, España, Inglaterra y Suiza, Santiago de Chile, Editorial Roberto Miranda, 1889. 
Bello, Andrés: Programa para los exámenes de los alumnos de la clase de principios generales de legislación: Colegio de Santiago, Santiago, Imprenta de la Opinión, 1831.

Briseño, Ramón: Estadística bibliográfica de la literatura chilena, Santiago de Chile, Imprenta Chilena, 1862.

Donoso, Ricardo: Las ideas políticas en Chile, México, Fondo de Cultura Económica, 1946.

Donoso, «José Joaquín de Mora y la Constitución de 1828», Revista Chilena de Historia del Derecho, 1, Santiago de Chile, 1959, 13-24.

Egaña, Juan: Memoria sobre los mayorazgos de Chile, de dos de junio de 1828, Santiago de Chile, Imprenta de R. Renjifo, 1828..

Labarca, Amanda: Historia de la enseñanza en Chile, Santiago de Chile, Imprenta Universitaria, 1939.

Meneses, Juan F.: Reglamento interior del Colegio de Santiago, Santiago, Imprenta R. Renjifo, 1829.

Meneses, Cuadro de ejercicios para cada día de la semana, Santiago, Imprenta R. Renjifo, 1829.

Mora, José J. de: Respuesta a la memoria sobre los mayorazgos de Chile publicada en Santiago, 2 de junio de 1828, Santiago de Chile, Imprenta de Renjifo, 1828.

Mora, «Curso de derechos del Liceo de Chile aplicable a todas las nuevas repúblicas de América», La Clave de Chile, periódico político y noticioso, N. 8, Santiago, Imprenta Republicana, 13 de agosto de 1829.

Mora, «Epistolario. Cartas de don José Joaquín de Mora a don Florencio Varela», Revista Chilena de Historia y Geografía, 50, Santiago de Chile, 1936, 35-66.

Mora, Plan de estudios del Liceo de Chile, con algunos pormenores sobre su ejecución y sobre la disciplina del establecimiento, Santiago, Imprenta R. Renjifo, 1828.

Poblete, Juan: «Literatura, discurso y legitimidad: la polémica de José Joaquín Mora y Andrés Bello», Estudios. Revista de Investigaciones Literarias y Culturales, 14-15, Caracas, 1999-2000, 145-171.

Salazar Vergara, Gabriel: Construcción de Estado en Chile. (1800-1837), Santiago de Chile, Editorial Sudamericana, 2007.

Serrano, Sol: Universidad y Nación, Santiago de Chile, Editorial Universitaria, 1994.

Silva Castro, Raúl: Fundación del Instituto Nacional (1810-1813), Santiago de Chile, Imprenta Universitaria, 1953.

Silva Castro, «José Joaquín de Mora y la Constitución de 1828», Revista de Historia de América, 43, Santiago de Chile, 1957, 109-113.

Stuardo Ortiz, Carlos: El Liceo de Chile. 1828-1831. Antecedentes para su historia, Santiago de Chile, Imprenta Universitaria, 1950. 
Valencia Avaria, Luis: Anales de la República, Santiago de Chile, Editorial Andrés Bello, 1986.

Verdugo Marinkovic, Mario y García Barcelatto, Ana María: Manual de derecho político. Instituciones políticas, Santiago de Chile, Editorial Jurídica de Chile, 2004.

Verdugo Marinkovic, Pfeffer Urquiaga, Emilio y Nogueira Alcalá, Humberto: Derecho constitucional, Santiago de Chile, Editorial Jurídica de Chile, 1994. 